\title{
Assessing Cerebrospinal Fluid Flow Dynamics in Pediatric Patients with Central Nervous System Tumors Treated with Intraventricular Radioimmunotherapy
}

\author{
Kim Kramer ${ }^{1}$, Maria Donzelli ${ }^{1}$, Emmanuela Nwora ${ }^{1}$, and Neeta Pandit-Taskar ${ }^{2}$ \\ ${ }^{I}$ Department of Pediatrics, Memorial Sloan Kettering Cancer Center, New York, New York; and ${ }^{2}$ Department of Radiology, Memorial \\ Sloan Kettering Cancer Center, New York, New York
}

\begin{abstract}
The incidence of abnormal cerebrospinal fluid (CSF) flow dynamics in children with central nervous system (CNS) tumors before intraventricular therapy has not been described. Methods: We performed a single-institution, retrospective review of patients with primary or metastatic CNS tumors treated between 2003 and 2018 (15 y). Patients underwent ${ }^{111} \mathrm{In}$-diethylenetriaminepentaacetic acid injection into the CSF intraventricular space followed by nuclear medicine imaging at $90 \mathrm{~min}, 4 \mathrm{~h}, 24 \mathrm{~h}$, and $48 \mathrm{~h}$ (if required). CSF flow was classified as normal, delayed, asymmetric, or obstructed. Results: In total, 278 CSF flow studies were performed on 224 patients, 202 of whom (90\%) were less than $18 \mathrm{y}$ old. Of these, 116 patients $(52 \%)$ had metastatic CNS neuroblastoma, $57(25 \%)$ had medulloblastoma, and $51(23 \%)$ had other histologic types of CNS tumors. Of the 278 studies, 237 (85\%) were normal, 9 (3\%) required neurosurgical intervention, 25 (9\%) were delayed, and $7(3 \%)$ were asymmetric. Conclusion: Abnormal CSF flow and the necessity for neurosurgical intervention must be considered when attempting to ensure appropriate intraventricular therapy in the pediatric population.
\end{abstract}

Key Words: cerebrospinal flow; leptomeningeal disease; radioimmunotherapy

J Nucl Med 2020; 61:662-664

DOI: 10.2967/jnumed.119.232678

$\mathbf{T}$ he success of intraventricular compartmental therapies, bypassing the blood-brain barrier, in part depends on adequate drug distribution throughout the thecal space. Several techniques may be used to assess cerebrospinal fluid (CSF) dynamics in the central nervous system (CNS), including phase-contrast and timespatial-labeling MRI. Radionuclide shuntography using ${ }^{99 m} \mathrm{Tc}-$ diethylenetriaminepentaacetic acid (DTPA) and serial imaging is another reliable way of evaluating CSF dynamics, although there is a general lack of guidelines on this procedure, as well as a lack of consensus on image interpretations (1). We undertook this study to define the incidence of normal, delayed, asymmetric, and obstructed CSF flow in a large pediatric population with CNS tumors before the administration of intraventricular therapy.

Received Jun. 18, 2019; revision accepted Sep. 16, 2019.

For correspondence or reprints contact: Kim Kramer, Department of Pediatrics, Memorial Sloan Kettering Cancer Center, 1275 York Ave., Box 429, New York, NY 10065.

E-mail: kramerk@mskcc.org

Published online Jan. 31, 2020.

COPYRIGHT (C 2020 by the Society of Nuclear Medicine and Molecular Imaging.

\section{MATERIALS AND METHODS}

We performed a retrospective review of patients with primary or metastatic CNS tumors treated at Memorial Sloan Kettering Cancer Center over a 15-y period (2003-2018). The patients were the subjects of Memorial Sloan Kettering Cancer Center institutional review board-approved protocol 16-1466, a study of the reliability of ${ }^{111} \mathrm{In}$ DTPA studies. Pediatric patients under consideration for trials incorporating radioimmunotherapy were required to have CSF flow studies documenting the absence of obstructive hydrocephalus and adequate CSF flow. All patients had an indwelling intraventricular catheter with either an Ommaya reservoir or a programmable ventriculoperitoneal shunt, with the ventricular location of the catheter confirmed on MRI or CT. The intraventricular catheter was accessed by the pediatric team (oncologist or nurse practitioner) in the presence of a nuclear medicine physician. The same clinician then used a 25 -gauge needle under sterile conditions; clear CSF was aspirated, confirming ventricular access. Approximately 7.4-11.1 MBq $(0.2-0.3 \mathrm{mCi})$ of ${ }^{111} \mathrm{In}-$ DTPA were injected into the ventricle, followed by an autologous CSF flush. Imaging was obtained using a Skylight dual-head $\gamma$-camera system (Philips Inc.). Whole-body conjugate (anterior, posterior, and bilateral) images of the head were acquired, as well as anterior and posterior images of the spine. The first nondynamic set of images was obtained within $90 \mathrm{~min}$ and up to $4 \mathrm{~h}$ after injection; this window was chosen to accommodate the pediatric patients, who often required general anesthesia for the scan, or to accommodate the capabilities of the scanner. Patients then underwent follow-up nuclear medicine imaging at $24 \mathrm{~h}$ and $48 \mathrm{~h}$ (if necessary). The distribution of the ${ }^{111} \mathrm{In}-$ DTPA was assessed on serial imaging and was officially read by a board-certified nuclear medicine physician and reviewed by the pediatric oncology team. CSF flow findings were classified as normal if tracer flowed down the thecal sac in the first scan and showed a distribution over the convexities and systemic clearance on the 24-h scan (Fig. 1A). CSF flow was considered obstructed if no tracer was seen down the thecal sac on any scan (Fig. 1B), asymmetric if one side of the brain had preferential uptake of tracer (Fig. 1C), and delayed if the tracer was slower to distribute over the convexities (Fig. 1D).

\section{RESULTS}

In total, $278 \mathrm{CSF}$ flow studies were performed on 224 patients, 202 of whom (90\%) were less than 18 y old; $116(52 \%)$ of these patients had a diagnosis of metastatic CNS neuroblastoma, 57 (25\%) had medulloblastoma, and 51 (23\%) had other histologic types of CNS tumors. There were no complications (i.e., extravasation, infection, or inability to acquire or remove CSF) associated with the administration of ${ }^{111}$ In-DTPA. In general, Ommaya catheters and ventriculoperitoneal shunts with programmable valves were equally easy to access, although a greater CSF volume 


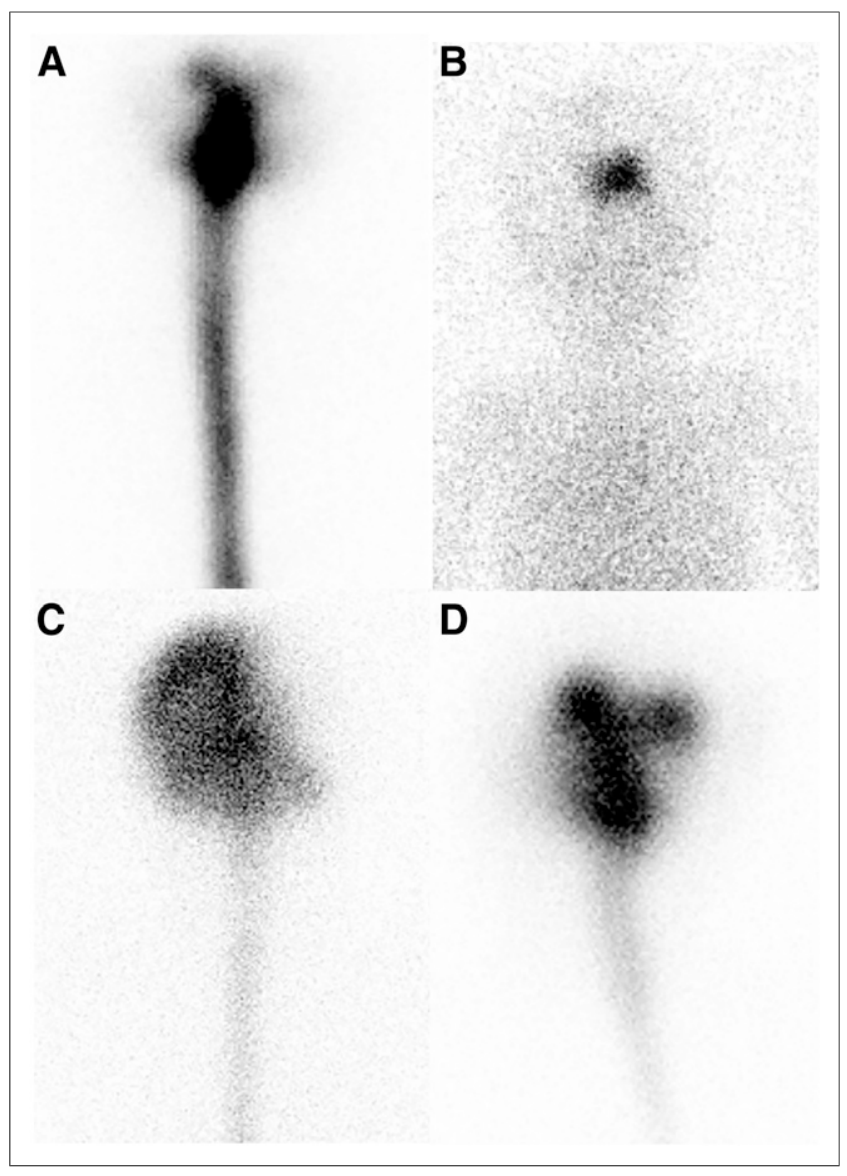

FIGURE 1. (A) Normal ${ }^{111}$ In-DTPA and nuclear medicine imaging results over $48 \mathrm{~h}$ in child with CNS neuroblastoma brain metastases. Patient was treated with surgery, radiation therapy, and chemotherapy and was in radiographic remission at time of this study, with no evidence of neuroblastoma on MRI. (B) Obstructed CSF flow-despite normal MRI results-seen at $24 \mathrm{~h}$ after injection in 3-y-old with relapsed medulloblastoma. Patient underwent endoscopic third ventriculostomy with lysis of adhesions, which reestablished adequate CSF flow and enabled treatment with radioimmunotherapy. (C) Asymmetric CSF flow shown by increased activity over right hemisphere on scan $20 \mathrm{~h}$ after injection in 8$y$-old boy with recent right parietal craniotomy and resection of metastatic lesions in right frontal lobe. MRI was unremarkable for evidence of hydrocephalus or subdural fluid collections. (D) Delayed CSF, with retention in ventricle at $24 \mathrm{~h}$ after injection, in 13-y-old boy with multiply recurrent medulloblastoma and known leptomeningeal metastases at time of injection. At $4 \mathrm{~h}$ after injection, images showed tracer accumulation predominantly in basilar cisterns and ventricles, with decreased flow down remainder of thecal sac.

for standard assays (i.e., cytology, cell count, and total protein levels) was obtainable from Ommaya catheters. In addition, administration of an Ommaya catheter did not require shunt valve adjustments, making them less cumbersome for both patients and staff. Further, despite full programming of a ventriculoperitoneal shunt at the highest setting (i.e., "off"), residual tracer activity was still visualized in the peritoneum at $24 \mathrm{~h}$ (Fig. 2). Of the 278 flow studies, 237 (85\%) were normal (Fig. 1A), 41 (15\%) were abnormal, 25 (9\%) were delayed (Fig. 1B), 9 (3\%) were obstructed and required neurosurgical intervention (Fig. 1D), and 7 (3\%) were asymmetric (Fig. 1C). Obstruction was evident for those scans that had no distribution down the thecal sac by $4 \mathrm{~h}$, with little or no change seen at 24 or $48 \mathrm{~h}$. Surgical correction to lyse adhesions or shunt revision to correct occlusion was required to establish flow in these instances.

For delayed scans, tracer resorption into the systemic circulation took more than $24 \mathrm{~h}$ and was evident on scans obtained at later intervals (i.e., $48 \mathrm{~h}$ ). The $24 \mathrm{~h}$ of drainage over the convexities is the rule for cisternograms (when lumbar injection is performed); in contrast, for Ommaya shunt studies, delayed flow was noted by decreased flow over the convexities at $24 \mathrm{~h}$ accompanied by decreased resorption into the systemic circulation.

An asymmetric distribution of tracer was evident on scans obtained at $24 \mathrm{~h}$, where one hemisphere had preferential uptake compared with the other. No surgical procedure was performed to address delayed or asymmetric CSF flow.

\section{DISCUSSION}

CSF dynamics have clinical, surgical, and therapeutic implications. For many years, contrast myelography was the procedure of choice to assess CSF flow, but contrast myelography was later replaced by radionuclide cisternography, particularly when detecting CSF fistulous tracts (2). Radionuclide imaging has often used 99mTc-DTPA or ${ }^{111}$ In-DTPA $(2,3)$. More recently applied has been phase-contrast MRI, a noninvasive imaging technique that enables quantitative measurements of CSF flow (4). Although this technique has been validated, a single injection of ${ }^{111} \mathrm{In}$-DTPA followed by serial images over 1-2 d provides additional therapeutic information by depicting the rate of CSF flow throughout the thecal space, the time to trace over the CNS convexities, and the time to achieve systemic absorption. These studies simulate the dynamics that may be predicted with radioimmunotherapy. Further, the use of ${ }^{111} \mathrm{In}$ DTPA imaging demonstrates the amount of therapeutic activity that may or may not be asymmetrically distributed, or lost in the peritoneal cavity as with VP shunts, with obvious therapeutic implications.

Several pathologic states are known to disrupt CSF flow dynamics, including the presence of bulk leptomeningeal deposits, postsurgical adhesions, and Chiari malformations (4). Recently, MRI studies have shown that anatomic differences, including lordosis and kyphosis, among healthy subjects affect CSF bulk flow; spinal cord eccentricity in the healthy human spine results in subject-specific patterns of recirculation of bulk CSF flow in certain regions (5).

Several methods to bypass

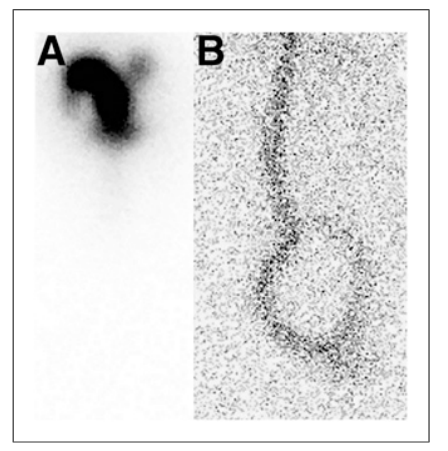

FIGURE 2. ${ }^{111}$ In-DTPA injection through programmable VP shunt. (A) Immediate-postinjection image shows CSF accumulation in basilar cisterns and ventricles, with no evidence of obstruction. (B) Four-hour images show minimal excretion from radiotracer-shunt catheter in peritoneum. the blood-brain barrier and improve the effectiveness of macromolecule drugs for CNS tumors have been attempted, with intraventricular administration of chemotherapy and radioimmunotherapy being among the most common. More recently, the study of interstitial fluid in the brain tissue and CSF, as well as the glia-lymphatic system and perivascular spaces for waste clearance, have been recognized as important links to the transport of solutes in the CNS $(6,7)$. Data further support the possibility that the perivascular spaces lining the leptomeninges provide an avenue of transport deep into 
the brain via CSF (8). Asymptomatic patients with no evidence of hydrocephalus on neuroradiographic imaging may have altered CSF flow dynamics that have therapeutic implications for intraventricular therapy.

Our results show that up to $15 \%$ of patients with CNS tumors have mechanisms that may affect the distribution of therapies in the CSF space, even in the absence of radiographic findings suggestive of altered CSF dynamics. This finding has therapeutic implications, because for treatments such as radioimmunotherapy, contact with the target antigen is necessary for radionuclide tumor ablation. Among these abnormal studies, there exist patients in whom no therapeutic drug would have been successfully distributed throughout the thecal space in the absence of a neurosurgical procedure to address flow dynamics. An asymmetric distribution of tracer is largely considered to be related to the presence of tumor deposits or to prior therapies resulting in adhesions or vascular insults (i.e., postsurgical or radiation therapy-induced changes), although innate interpatient variability is still possible. These results support baseline isotope tracer studies for any patient in whom intraventricular therapies are being considered, not only for malignancies but for several other neurodegenerative conditions with proposed therapies circumventing the blood-brain barrier (i.e., amyotrophic lateral sclerosis, Alzheimer and Parkinson diseases, and lysosomal storage diseases).

\section{CONCLUSION}

Abnormal CSF flow studies were noted in $15 \%$ of pediatric patients with CNS tumors, including $3 \%$ of patients who would require a procedure to improve adequate flow before receiving intraventricular therapy. These results have implications for successful delivery of intraventricular therapies.

\section{DISCLOSURE}

Kim Kramer is a consultant to Ymabs Therapeitucs, Inc. This work was supported by NCI Cancer Center support grant P30 CA008748. No other potential conflict of interest relevant to this article was reported.

\section{ACKNOWLEDGMENT}

We thank Joe Olechnowicz for editorial assistance in submitting the article.

\section{KEY POINTS}

QUESTION: What is the incidence of normal, delayed, asymmetric, and obstructed CSF flow in the pediatric population with CNS tumors before intraventricular radioimmunotherapy?

PERTINENT FINDINGS: Abnormal CSF flow results were found for $15 \%$ of pediatric patients with CNS tumors; $3 \%$ of patients would require a procedure to improve adequate flow before receiving intraventricular therapy.

IMPLICATIONS FOR PATIENT CARE: Because adequate CSF flow is important to the successful therapeutic use of chemotherapy and radioimmunotherapy, oncologists should consider demonstrating a normal distribution before performing these interventions.

\section{REFERENCES}

1. Tsai SY, Wang SY, Shiau YC, Yang LH, Wu YW. Clinical value of radionuclide shuntography by qualitative methods in hydrocephalic adult patients with suspected ventriculoperitoneal shunt malfunction. Medicine (Baltimore). 2017;96: e6767.

2. Krasnow AZ, Collier BD, Isitman AT, Hellman RS, Joestgen TM. The use of radionuclide cisternography in the diagnosis of pleural cerebrospinal fluid fistulae. J Nucl Med. 1989;30:120-123.

3. Rosen PR, Chaudhuri TK. Radioisotope myelography in the detection of pleuraldural communication as a source of recurrent meningitis. Clin Nucl Med. 1983; 8:28-30.

4. Sakhare AR, Barisano G, Pa J. Assessing test-retest reliability of phase contrast MRI for measuring cerebrospinal fluid and cerebral blood flow dynamics. Magn Reson Med. 2019;82:658-670.

5. Coenen W, Gutierrez-Montes C, Sincomb S, et al. Subject-specific studies of CSF bulk flow patterns in the spinal canal: implications for the dispersion of solute particles in intrathecal drug delivery. AJNR Am J Neuroradiol. 2019;40:12421249.

6. Abbott NJ, Pizzo ME, Preston JE, Janigro D, Thorne RG. The role of brain barriers in fluid movement in the CNS: is there a 'glymphatic' system? Acta Neuropathol (Berl). 2018;135:387-407.

7. Pizzo ME, Wolak DJ, Kumar NN, et al. Intrathecal antibody distribution in the rat brain: surface diffusion, perivascular transport and osmotic enhancement of delivery. J Physiol (Lond). 2018;596:445-475.

8. Papisov MI, Belov VV, Gannon KS. Physiology of the intrathecal bolus: the leptomeningeal route for macromolecule and particle delivery to CNS. Mol Pharm. 2013;10:1522-1532. 